\title{
Der gemachte Mensch?!
}

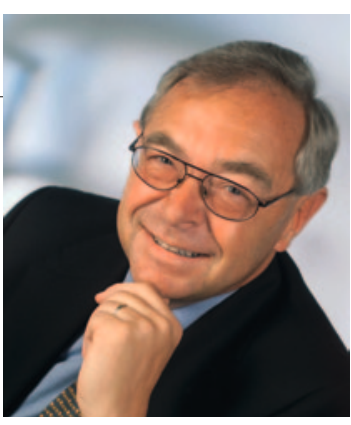

HERAUSGEBER

Univ.-Prof.

Dr. Norbert Bachl

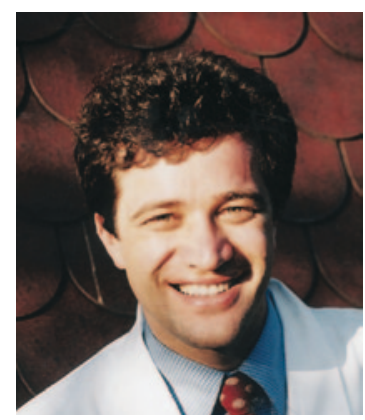

EDITOR IN CHIEF

Dr. Piero Lercher

Wer zahlt die Kosten, wenn nach Schönheitsoperationen Komplikationen auftreten, welche weiter behandelt werden müssen und den Sozial- und Krankenversicherungen Kosten verursachen? Wer zahlt die Kosten, wenn verschiedene Hormonpräparate Nebenwirkungen nach sich ziehen, welche eine weitere diagnostische und therapeutische Intervention benötigen? Wer zahlt die Kosten, wenn Gelenke, Sehnen, Knorpel, Bänder einen bis an die Schmerzgrenze gesteigerten Trainingsprozess nicht aushalten bzw. innere Organe durch - zwar verbotene, aber oft benutzte - Drogen schwerst geschädigt werden? Wer zahlt die Kosten, wenn junge Menschen einer Schönheitsillusion folgend in die Anorexie abgleiten und behandelt werden müssen?

Viele Fragen, welche in der vorliegenden Ausgabe des Journals für Sport- und Präventivmedizin nicht beantwortet werden können - dazu müssten ein interdisziplinäres Team vom Psychiater bis zum Endokrinologen und ein Vielfaches der Seitenzahl dieses Heftes zur Verfügung stehen. Allerdings soll das Editorial zum Nachdenken anregen. Darüber hinaus sollten wir Mediziner uns mit diesen immer öfters thematisierten Fragen beschäftigen, da eine größer werdende Patienten- bzw. Klientenzahl Fragen stellt. In diesem Sinn sind auch die Beiträge zu verstehen, welche für die Praxis wesentliche Hinweise geben.

In der aktuellen Ausgabe der sport- und präventivmedizin setzen wir auch wieder unsere beliebten Serien, wie beispielsweise die Ernährungsmärchen, fort und bieten Ihnen wieder praxisrelevante Doc's Tipps und interessante Fallstricke. Im Panorama finden Sie eine Auswahl von interessanten Veranstaltungen und empfehlenswerten Kongressen.

Wir wünschen Ihnen, sehr geehrte Leser und Leserinnen, viel Spaß beim Lesen! Herzlichst,

\author{
Univ.-Prof. Dr. Norbert Bach \\ Herausgeber
}

\author{
Dr. Piero Lercher \\ Editor in Chief
}

